\title{
Various Roadside Populations Effects on Data Throughput over Cooperative Vehicular Ad hoc Networks
}

\author{
Esraa Eldesouky ${ }^{1},{\text { Ahmed } \mathrm{Ali}^{1}, \mathrm{Li} \mathrm{Renfa}^{1} \text { and Wang Dong }}^{1}$ \\ ${ }^{1}$ College of Information Science and Engineering,Hunan University,410082, \\ Changsha, China \\ esraa_m@hnu.edu.cn
}

\begin{abstract}
Roadside units in Vehicular Ad hoc Networks are essential for acting as base stations that facilitate information spreading to vehicular users in the roads. Typically, vehicular nodes search for an infrastructure within their vicinity to fulfill the requested services such as internet accessing or content file handling. Due to the essentiality of the presence of roadsides as well as the high costs for installing them, determining the appropriate roadside populations is an ongoing challenge. According to the surrounding vehicular density, we aim to assure higher data throughput in order to accomplish a cooperative network. This paper considers the roadside populations as to supply vehicles' requirements based on both a proposed algorithm and simulation results. Moreover, we study the roadside selfparticipation to enhance the transfer rates and achieve fair resource distribution among the requested vehicles based on coalition formation. Simulation results have proven that the variation of the roadside populations influences the data throughput. Additionally, the cooperative environment among vehicular nodes achieves higher roadside participation ratios especially in the maximum case.
\end{abstract}

Keywords: Vehicular Ad hoc Networks; Data Throughput; Roadside Participation Percentage; Coalition Formation

\section{Introduction}

Wireless technologies have been adopted by vehicle manufacturers since 1980s, which results in the Vehicular Ad hoc Networks (VANETs) [1-3]. Communications over VANETs include Vehicle-to-Vehicle (V2V) and Vehicle-to-Roadside (V2R). Researchers aim to achieve a full wireless communication environment among vehicular components. Achieving these requirements is mainly depending on collecting real-time data on road conditions and put them in useful form through a wide range of applications including safety and comfortable applications [4].

Several applications are provided by Roadside Units (RSUs) installed along the roads. These units are wave devices fixed along areas such as junctions or parking spaces. They are equipped with network devices for Dedicated Short Range Communications (DSRC) based on IEEE $802.11 \mathrm{p}$ radio technology and other devices used for communications within different networks [5]. RSUs can perform as information distributor, Internet provider, and safety applications supporter [6].

RSU placement problems have been addressed in several works as in [7-10]. In [7], the authors used three allocation methods including: 1) the most driving routes first, 2) the most satisfied intersection pairs, 3) the critical intersections first. This study is a NP-hard problem [11] which shows that the most satisfied intersections and the critical intersection first 
methods yields to lower number of the required RSUs than the most driving routes. An analytical model to estimate the minimum number of the required RSUs was proposed in [8], where the packets delay between the vehicles and RSUs are bounded. However in [9], the RSU settlement problem which minimizes the disconnection time between vehicles and RSUs are studied and a placement scheme was introduced. Another RSU deployment scheme is presented in [10]. The authors aim to know where to place RSUs as to cover a maximum number of vehicles. This problem is modelled as a Maximum Coverage with Time Threshold Problem (MCTTP), and a genetic algorithm is used to solve this problem.

In this paper, we focus on determining suitable roadside populations according to a given vehicular node densities. Our model intends to maximize the data throughput through achieving better resource managements, specifically bandwidth utilization. Coalition formation game is used to analyze the roadside participation ratio of several roadside populations and their impact on requesting vehicles within their coverage; along with achieving higher satisfaction rates to the vehicular nodes. Finally, the first coalition formation approach for bandwidth sharing in VANETs was presented in [12]. Authors consider the cooperation among vehicular components in $\mathrm{V} 2 \mathrm{R}$ environment in order to maximize the total network utility without considering the individual payoffs.

The rest of this paper is organized as follows: A problem explanation and a descriptive flow chart are presented in Section 2. In Section 3, the coalition formation model is introduced along with the proposed algorithm. We list the performance metrics and evaluations for efficiency measurements in Section 4. Section 5 includes the experimental results and discussions for the different experiments. Finally, Section 6 concludes our paper.

\section{Problem Description}

In VANETs, enormous information is exchanged can be requested from the RSUs [13]. Although V2R communications can supply users' needs, some problems still occur due to high mobility and dynamic topology. 1) Uneven distribution of RSUs resources among vehicles. 2) The difficulty of maintaining end-to-end connection between RSUs and requested vehicles due to the fragile links. 3) The limitation of RSUs resources which lead to the presence of competitive and rational nodes thus degrades the network throughput. According to the mentioned problems, we formulate the RSU access problem in a coalition formation game for studying the impact of RSU populations for a given number of requested vehicles (see Figure 1). An important question that we investigate is whether the number of roadside populations in a given area affect data throughput. This study mainly intends to reduce RSUs installation costs by studying the participation ratio for different roadside populations.

Generally, vehicles are controlled by bound self-interested entities (i.e., humans) [13] These entities can seldom take part in resource sharing process as they selfishly seek for reserving their needs first. Consequently, stimulating these nodes to attain better individual payoffs along with the overall network gain is necessary. In this proposed model, our vehicular nodes are equipped with appropriate network devices for accessing the IEEE 802.11p wireless channels and facilitate the V2R communication. Likewise, Global Positioning System (GPS) devices are mounted to vehicles to detect other nodes' locations. It is a processing model which includes a set of vehicles and roadsides as an input set, and a set of formed coalitions as an output set. Last but not least, the processing operation is defined to be the efficient bandwidth allocation to achieve maximum overall gain and enhance the network throughput. 


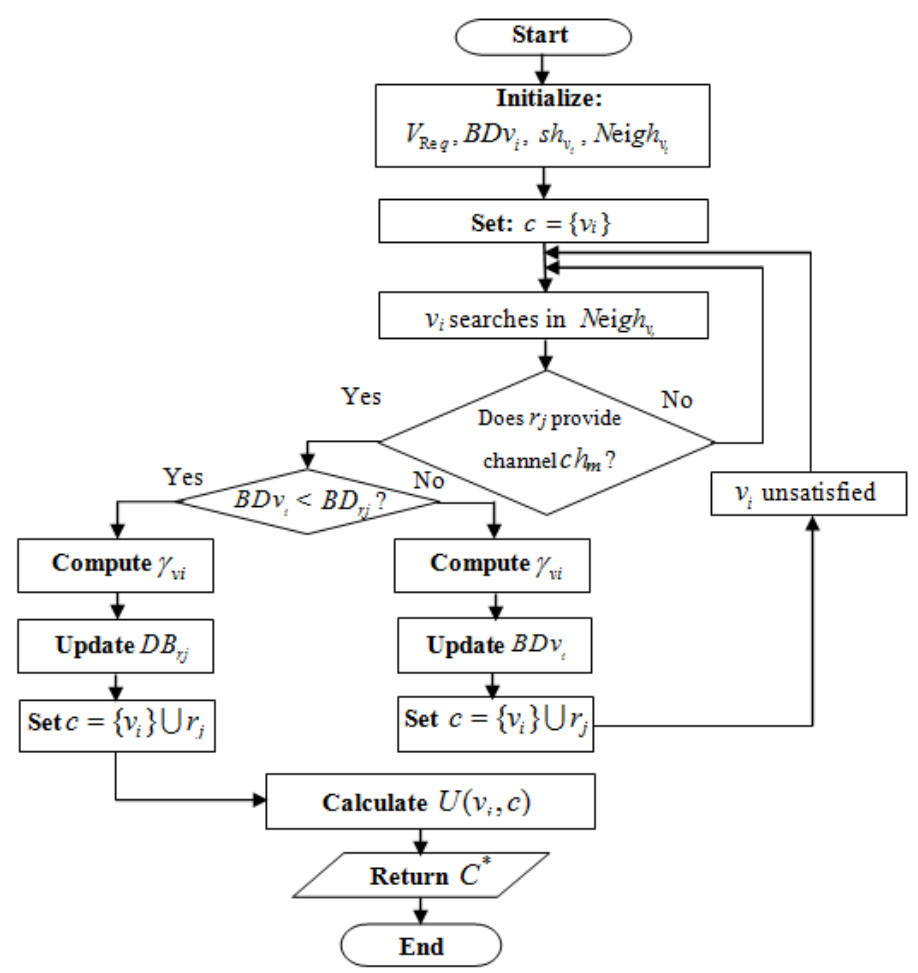

Figure 1. The Flowchart of the Proposed Algorithm

\section{Coalition Formation Approach}

\subsection{Model Notation}

The proposed model $G$ is represented as $G=<V, R, c h_{i}, U_{i}>$, such that $V=\left\{v_{1}, \ldots, v_{n}\right\}$ and $R=\left\{r_{1}, \ldots, r_{m}\right\}$ are sets of vehicles and RSUs respectively. Each vehicle $v_{i} \in V$ can cooperate with other vehicular nodes within its vicinity as to acquire the demanded resources. These nodes communicate with each other through a set of non-overlapping wireless channels $C H=\left\{c h_{1}, \ldots, c h_{l}\right\}$. Our main goal is to cooperatively enhance bandwidth utilization. Thus, better data transmission rates can be achieved along with improving vehicle's satisfaction rate. Table 1 summarizes the variables used in our coalition formation model.

Vehicles usually search for the nearest roadside $r_{j}$ for $r_{j} \in R$ in order to form cooperative set of coalitions $C$, where $C=\left\{c_{1}, \ldots, c_{x}\right\}$. These vehicles are able to form a set of $x$ possible coalitions such that $x \in 2^{v_{n}}-1$. For any coalition $c_{y}$ (i.e., $\left.y=1, \ldots, x\right)$, is defined to be a nonempty set of $V$ and $R$ in which $R$ provides the requested bandwidth to $V$ according to the desired channel. Based on the needed channel, a demanded vehicle $v_{i}$ will afford a cost $\gamma_{v_{i}}$ for reserving such a resource from roadsides within its coverage area. Consider the RSUs as a bandwidth provider, a constant price $\theta$ is computed where $\theta=0.3 / \sigma$ for $\sigma=27 \mathrm{~KB}$ ps (i.e., the transmission rate per unit for bandwidth $\beta)$. Consequently, the $\operatorname{cost} \gamma_{v_{i}}\left(c_{y}, c h_{m}\right)$ is defined 
to be the price consumed by a given vehicle $v_{i}$ for forming a coalition $c_{y}$ to access the requested channel $c h_{m}$, and is calculated according to equation (1):

$$
\gamma_{v_{i}}\left(c_{Y}, c h_{m}\right)=\beta_{v_{i}} \times \theta \quad \forall v_{i} \in c_{y} \text { and } c h_{m} \in C H
$$

Where $\gamma_{v_{i}}\left(c_{y}, c h_{m}\right) \in \square^{+}$.

In game theory, the utility function often estimates the preferences of each player in the formed coalition. The main target is to maximize the player's own expected payoff $[14,15]$. In our model, each vehicle $v_{i}$ vies for a permitted share value $s h_{v_{i}}$ of the value of the formed coalition, such that $s h_{v_{i}} \in \square^{+}$. Vehicles can obtain this share value by acquiring the requested bandwidth and achieve its level of satisfaction. Based on this share value $s h_{v_{i}}$ for a given vehicle $v_{i}$ and the incurred $\operatorname{cost} \gamma_{v_{i}}\left(c_{y}, c h_{m}\right)$ for joining the coalition $c_{y}$, our utility function is computed as in equation (2):

$$
U\left(v_{i}, c_{y}\right)=s h_{v_{i}}-\gamma_{v_{i}}\left(c_{y}, c h_{m}\right) \quad \forall v_{i} \in c_{y}, c_{y} \in C \text { and } c h_{m} \in C H
$$

The overall goal is to find a coalition structure such that vehicles are stimulated to join for increasing their individual utility. Therefore, the stable coalition $C^{*}$ with the maximum rewards indicates calculating the largest utility values by using the following equation:

$$
C^{*}=\arg \max \sum_{v_{i} \in c_{y}} U\left(\mathrm{v}_{i}, \mathrm{c}_{y}\right)
$$

The sum of the accomplished utilities for vehicles cooperated and formed feasible coalitions are computed in equation (3). Nonetheless, we are concerned with determining the percentage of the roadsides taking part in the coalition formation game as discussed in the following sections.

Table 1. Variables Description

\begin{tabular}{|l|l|}
\hline Parameters & Description \\
\hline$V$ & Set of vehicles \\
\hline$V_{\operatorname{Re} q}$ & Set of requested vehicles \\
\hline$R$ & Set of roadsides \\
\hline$C H$ & Set of wireless channels \\
\hline$C$ & Set of formed coalitions \\
\hline$\theta$ & The allocated bandwidth cost price \\
\hline$\sigma$ & Transmission rate per bandwidth unit (KBps) \\
\hline$B D v_{i}$ & The needed bandwidth for vehicle $v_{i} v_{i}$ \\
\hline$N e i g h_{v_{i}}$ & List of direct neighbors of $v_{i}$ \\
\hline$B D r_{j}$ & The available bandwidth at vehicle $v_{i}$ \\
\hline
\end{tabular}

\subsection{The Proposed Algorithm}

In the recent years, a variety of Intelligent Transportation Systems (ITS) applications have been developed to increase the transportation system performance by reducing congestion, enhancing the safety, and supporting comfortable traveling [16]. This can be achieved by exchanging warning messages, or accessing multimedia contents. Accordingly, communications in VANETs are maintained through vehicles within each other or vehicles to 
roadsides. Thus, the proposed algorithm aims to improve network scalability, resource sharing and management, and reducing the cost of installing vehicular equipment. Vehicles prefer V2R communications on the road especially when requesting distinctive services that are not afforded by neighboring vehicles. However, the channel condition in V2R communications is less in quality than in $\mathrm{V} 2 \mathrm{~V}$. The reason behind that is the large number of vehicular nodes to be served by RSUs simultaneously, the short connection duration, and limitation of V2R communication period [17]. Through the proposed model, vehicles can efficiently utilize the available bandwidth reside at the roadsides within their coverage.

As in Figure 1, the algorithm is initialized by defining the set of requested vehicles $V_{\mathrm{Re} q}$ along with the needed bandwidth $B D_{v_{i}}$ for each vehicle $v_{i}$. In Phase 1 , we allocate a share value $s h_{v_{i}}$ for each vehicle $v_{i}$ and define the direct neighbor list for this vehicle. A coalition $c_{y}$ commences as a standalone coalition including the requested vehicle $v_{i}$.

\section{Phase 1: Initialization}

1. A set of requested vehicles $V_{\mathrm{Re} q}=\left\{v_{1}, \ldots, \mathrm{v}_{n}\right\}$ where $v_{i} \in V$

2. For each vehicle $v_{i}$ assign a needed bandwidth $B D_{v_{i}}$ for the channel $c h_{m}$ and a share value $s h_{v_{i}}$

3. Add $v_{i}$ to a coalition $c_{y}$

4. Set Neigh $h_{v_{i}}$ as the neighbor list of $v_{i}$

The algorithm in the Searching Process phase is obtained by searching in the direct neighbor list $\mathrm{Neigh}_{v_{i}}$ for a roadside that can afford the bandwidth demanded by vehicle $v_{i}$. Whenever there is a roadside $r_{j}$ in the direct neighbor list Neigh $h_{v_{i}}$ that can partially supply the needed bandwidth $B D_{v_{i}}$, it is added to the coalition $c_{y}$. Nevertheless, the vehicle $v_{i}$ is counted as a requesting vehicle when the bandwidth $B D_{v_{i}}$ is not attained. In this case, the searching process continues to include the indirect neighbors of $v_{i}$.

\section{Phase 1: Searching Process}

For each $v_{i}$, search in the $N e i g h_{v_{i}}$ for the nearest $r_{j}, \forall r_{j} \in R$

If ( $r_{j}$ supports $\left.c h_{m}\right)$, then

Add $r_{j}$ to coalition $c_{y}$

Step 1: If $\left(B D r_{j} \geq B D_{v_{i}}\right)$

1. Compute the price cost $\gamma_{v_{i}}\left(c_{y}, c h_{m}\right)$, according to equation (1)

2. Update $B D_{r_{j}}$ as follow: $B D_{r_{j}}=B D_{r_{j}}-B D_{v_{i}}$

3. Consider $v_{i}$ as a satisfied vehicle, go to Utility Calculation phase.

Step 2: If $\left(B D_{r_{j}}<B D_{v_{i}}\right)$

1. Compute the price cost $\gamma_{v_{i}}\left(c_{y}, c h_{m}\right)$, according to equation (1)

2. Update $B D_{v_{i}}$ as follow: $B D_{v_{i}}=B D_{v_{i}}-B D_{r_{j}}$

3. Consider $v_{i}$ as an satisfied vehicle, go to Step 1 .

Else

Vehicle $v_{i}$ search in Neig $_{v_{i}}$ for another roadside 
As illustrated in Phase 2, a requested vehicle $v_{i}$ can satisfy the needed resources from the first level (i.e., direct neighbors) or from searching in further levels to include the indirect neighbors within its transmission range. A vehicle $v_{i}$ is said to be satisfied, when it succeeds to allocate the needed resource during the limited connection time with other vehicular nodes in its vicinity. At this stage, the utility for each vehicle participates in a coalition is computed and the total maximum reward of forming feasible coalition is returned.

\section{Utility Calculation Phase}

1. Calculate $U\left(v_{i}, c_{y}\right)$ according to equation (2).

2. Stop search and return the maximum reward $C^{*}$

Unlike other research work proposed for coalition formation in VANETs [12, 17-19], we intend the IEEE 802.11p standard that is mainly developed for VANETs [20]. The proposed model studies the enhancement of the individual node payoff by increasing the data throughput. This is achieved by allocating the demanded bandwidth or the needed resources efficiently. Moreover, the balanced occupations of the wireless channels will improve the network throughput by maximize the data transfer rates.

\section{Performance Metrics}

The efficiency of the proposed approach is analyzed through a wide range of experiments. Our simulation is built using Java programming language and the experiments are executed on a machine with $2.2 \mathrm{GHz}$ CPU, $8 \mathrm{~GB}$ RAM and windows 7 64-bits specifications. Each experiment contains a set of vehicles and roadsides, a maximum number of direct neighbors, and a set of channels and requested bandwidths assigned to each vehicular node. A total of 20 experiments are conducted based on four different vehicular densities assigned to a predefined number of neighbors divided into 2 regions. However, we change the number of the installed roadsides for each experiment to study the impact of their variation. Moreover, each of these experiments is executed 30 times; afterwards the maximum, minimum, and average results are taken to ensure accuracy of the proposed algorithm (See Table 2). Our experiments are limited to 40 vehicular nodes because of the machine's limited specifications.

Table 2. Experimental Parameters

\begin{tabular}{|c|c|c|c|c|}
\hline Experimental Area & \multicolumn{2}{|c|}{ Region 1 } & \multicolumn{2}{c|}{ Region 2 } \\
\hline Vehicular Density & 10 & 20 & 30 & 40 \\
\hline Direct Neighbors & 2 & 4 & 6 & 8 \\
\hline RSU Populations for each experiment & \multicolumn{3}{c|}{$1-3-5-7-9$} \\
\hline
\end{tabular}

\subsection{Data Throughput}

In general, the data throughput is vital in computer networks especially for bandwidth utilization. It is the successful departure rate for transferred data over a communication channel. Another definition for the data throughput according to [21] is defined to be "the average number of bits per second passed up from the MAC sublayer at the destination". This essential metric may be affected by different factors; one of the main factors is the end-user behavior. Mainly, we aim to stimulate the individual 
vehicles to take part in the coalition formation game. Firstly, the total number of successful channels allocation $(S A)$ is calculated as in the following equation:

$$
S A=\sum_{i=1}^{S_{c h}} V S_{i}
$$

Where $S_{c h}$ is the total number of the requested channels that are successfully allocated; and $V S$ is the number of vehicles that succeed to access the desired channel. The average data throughput metric in our model is obtained by using equation (5):

$$
A T=\frac{1}{\tau}\left(\sum_{j=1}^{x} S A_{j}\right)
$$

Where $\tau$ is the time required in seconds to allocate the requested channel, and $x$ is the total number of the formed feasible coalitions in set $C$.

\subsection{Roadside Participation Ratio}

As mentioned above, this paper targets the roadside installation ratio according to the surrounding vehicular nodes. As a result, the roadside participation ratio measures the number of the RSUs that take part in the coalition formation game; it can be calculated as follow:

$$
\begin{aligned}
R P R & =\sum_{i=1}^{\text {total }_{R}} P R_{i} \\
A_{R P R} & =\frac{R P R}{\text { total }_{R}} \times 100
\end{aligned}
$$

Where total $_{R}$ represents the total roadside populations in each experiment. $P R$ is the roadside that succeeds to join a formed coalition by providing the requested bandwidth.

\section{Simulation Results and Discussion}

In this section, we compare through simulation the effect of roadside on data throughput plus estimating their participation percentage within the formed coalitions. Based on the conducted experiments, the simulation results are listed in Table 3 and 4.

According to the metrics presented above, Figures 2, 3, 4, and 5 illustrate the numerical results specified in Tables 3 and 4. In Figure 2, minimum, average, and maximum results for data throughput and roadside participation percentages for 10 vehicular nodes are presented. According to Figure 2(a), vehicles succeed to achieve higher data throughput when 1 and 5 roadsides are installed. Generally, the less roadside population will reduce the installation expenses. The roadside participation percentage for the same experiment is shown in Figure 2(b).

Table 3. Data Throughput for Various Roadside Populations

\begin{tabular}{|c|c|c|c|c|c|}
\multicolumn{2}{|c|}{ Kehicles } & \multicolumn{2}{|c|}{ Region 1 } & \multicolumn{2}{c|}{ Region 2 } \\
\cline { 3 - 6 } \multicolumn{2}{|c|}{ RSUs } & 10 & 20 & 30 & 40 \\
\hline \multirow{3}{*}{1} & Min & 0.108 & 0.511 & 0.122 & 0.141 \\
\cline { 2 - 6 } & Max & 1.781 & 1.469 & 1.463 & 1.230 \\
\cline { 2 - 6 } & Avg. & 1.061 & 0.914 & 0.863 & 0.749 \\
\hline \multirow{2}{*}{3} & Min & 0.154 & 0.265 & 0.371 & 0.237 \\
\cline { 2 - 6 } & Max & 1.793 & 1.403 & 1.088 & 1.259 \\
\hline
\end{tabular}




\begin{tabular}{|c|c|c|c|c|c|}
\hline & Avg. & 0.884 & 0.723 & 0.881 & 0.695 \\
\hline \multirow{3}{*}{5} & Min & 0.413 & 0.319 & 0.277 & 0.323 \\
\cline { 2 - 6 } & Max & 1.594 & 1.045 & 1.100 & 0.905 \\
\cline { 2 - 6 } & Avg. & 0.988 & 0.726 & 0.695 & 0.628 \\
\hline \multirow{3}{*}{7} & Min & 0.188 & 0.442 & 0.284 & 0.539 \\
\cline { 2 - 6 } & Max & 2.333 & 1.152 & 1.060 & 0.999 \\
\cline { 2 - 6 } & Avg. & 0.853 & 0.831 & 0.763 & 0.775 \\
\hline \multirow{3}{*}{9} & Min & 0.298 & 0.577 & 0.338 & 0.349 \\
\cline { 2 - 6 } & Max & 1.581 & 1.328 & 1.005 & 1.091 \\
\cline { 2 - 6 } & Avg. & 0.790 & 0.892 & 0.693 & 0.734 \\
\hline
\end{tabular}

Table 4. Roadside Participation Percentage for the Two Regions

\begin{tabular}{|c|c|c|c|c|c|}
\hline \multirow{2}{*}{\multicolumn{2}{|c|}{$\begin{array}{l}\text { Kehicles } \\
\text { RSUs }\end{array}$}} & \multicolumn{2}{|c|}{ Region 1} & \multicolumn{2}{|c|}{ Region 2} \\
\hline & & 10 & 20 & 30 & 40 \\
\hline \multirow{3}{*}{1} & Min & $33.30 \%$ & $33.33 \%$ & $44.44 \%$ & $33.33 \%$ \\
\hline & Max & $100.00 \%$ & $100.00 \%$ & $75.00 \%$ & $75.00 \%$ \\
\hline & Avg. & $86.00 \%$ & $67.80 \%$ & $56.83 \%$ & $53.52 \%$ \\
\hline \multirow{3}{*}{3} & Min & $40.00 \%$ & $50.00 \%$ & $20.00 \%$ & $46.88 \%$ \\
\hline & Max & $100.00 \%$ & $85.71 \%$ & $80.95 \%$ & $72.22 \%$ \\
\hline & Avg. & $77.39 \%$ & $62.01 \%$ & $57.29 \%$ & $60.63 \%$ \\
\hline \multirow{3}{*}{5} & Min & $50.00 \%$ & $42.86 \%$ & $44.44 \%$ & $42.86 \%$ \\
\hline & Max & $100.00 \%$ & $86.67 \%$ & $80.00 \%$ & $77.78 \%$ \\
\hline & Avg. & $90.05 \%$ & $60.69 \%$ & $60.18 \%$ & $59.98 \%$ \\
\hline \multirow{3}{*}{7} & Min & $33.30 \%$ & $50.00 \%$ & $45.00 \%$ & $47.22 \%$ \\
\hline & Max & $100.00 \%$ & $75.00 \%$ & $70.00 \%$ & $75.00 \%$ \\
\hline & Avg. & $78.13 \%$ & $62.51 \%$ & $60.28 \%$ & $57.54 \%$ \\
\hline \multirow{3}{*}{9} & Min & $33.33 \%$ & $50.00 \%$ & $48.00 \%$ & $52.63 \%$ \\
\hline & Max & $100.00 \%$ & $100.00 \%$ & $70.73 \%$ & $66.67 \%$ \\
\hline & Avg. & $84.92 \%$ & $64.24 \%$ & $58.20 \%$ & $59.27 \%$ \\
\hline
\end{tabular}

Using 20 vehicles, a data transfer rate of $0.9 \mathrm{Mbps}$ is recorded as the highest average rate when the formed coalition is based on 1 roadside (see Figure 3(a)). Similarly in Figure 3(b), we notice that the average participation percentage when setting the roadside population to 1 can reach $67.8 \%$. This means that vehicular nodes can rely on the installed roadside to fulfill over $50 \%$ of the needed services. 


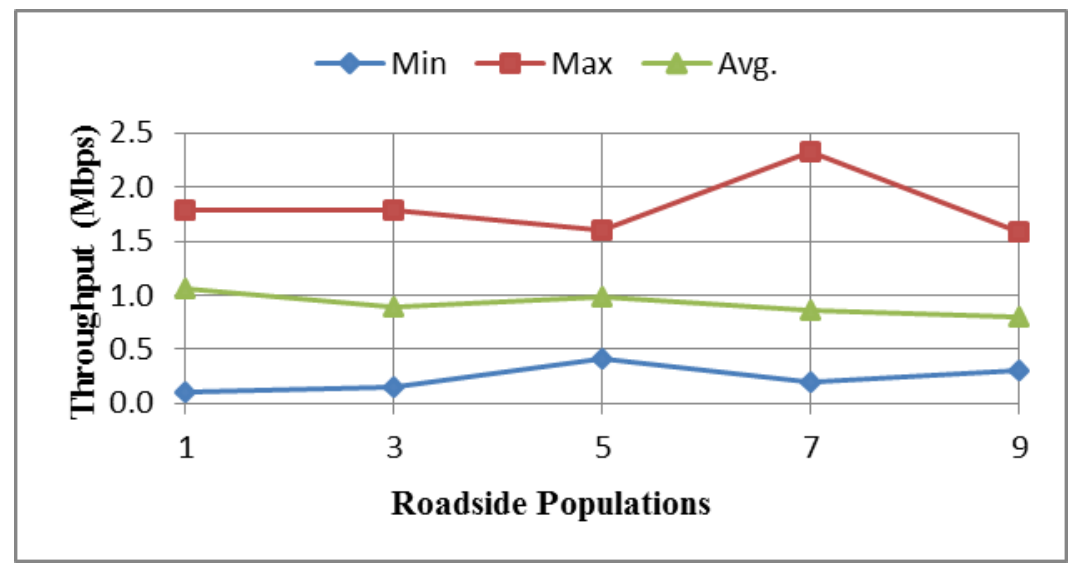

(a)

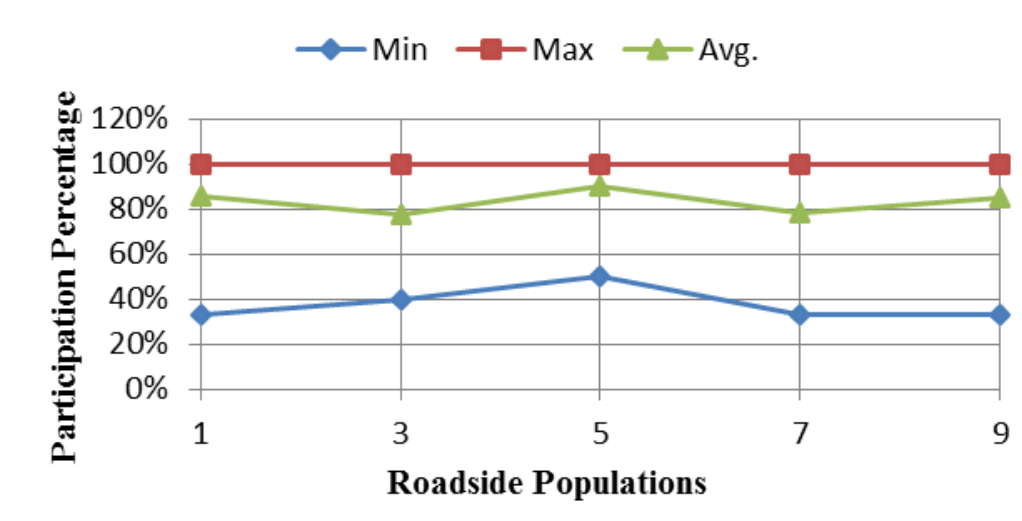

(b)

Figure 2. Throughput and Roadside Participation Percentage for 10 Vehicles

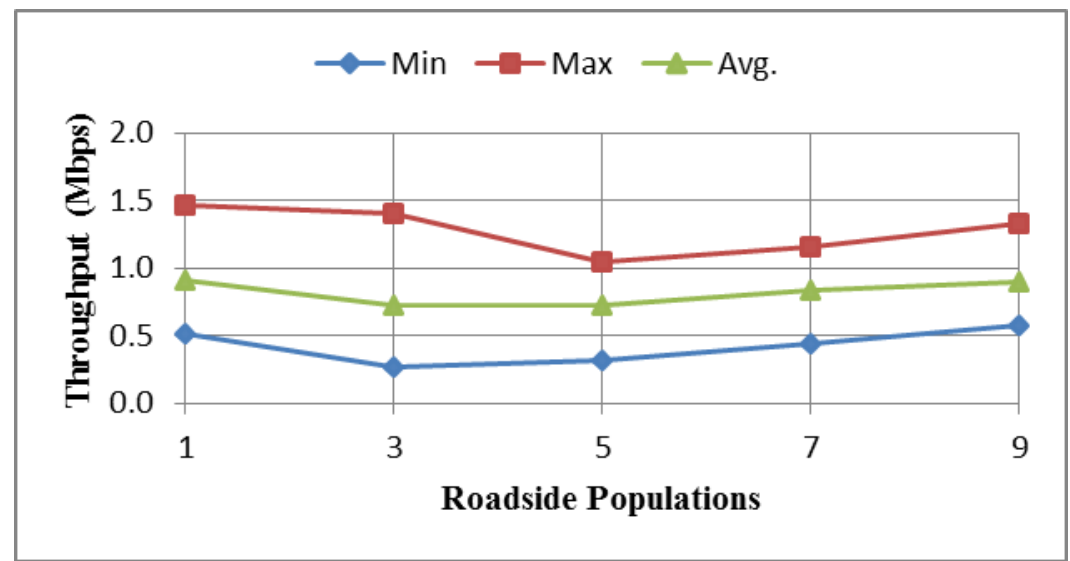

(a) 


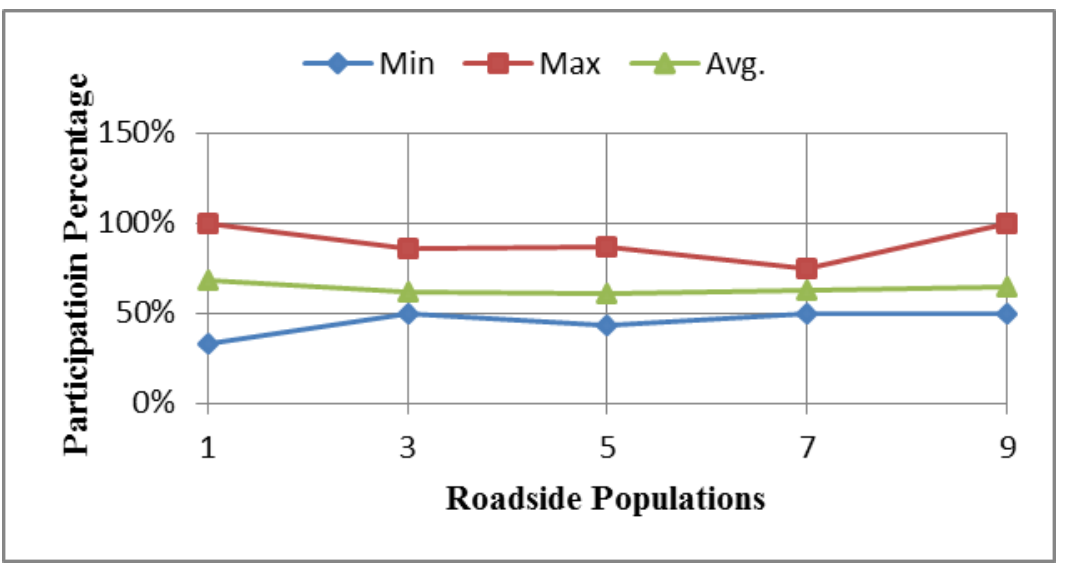

(b)

Figure 3. Numerical Results when Using 20 Vehicles

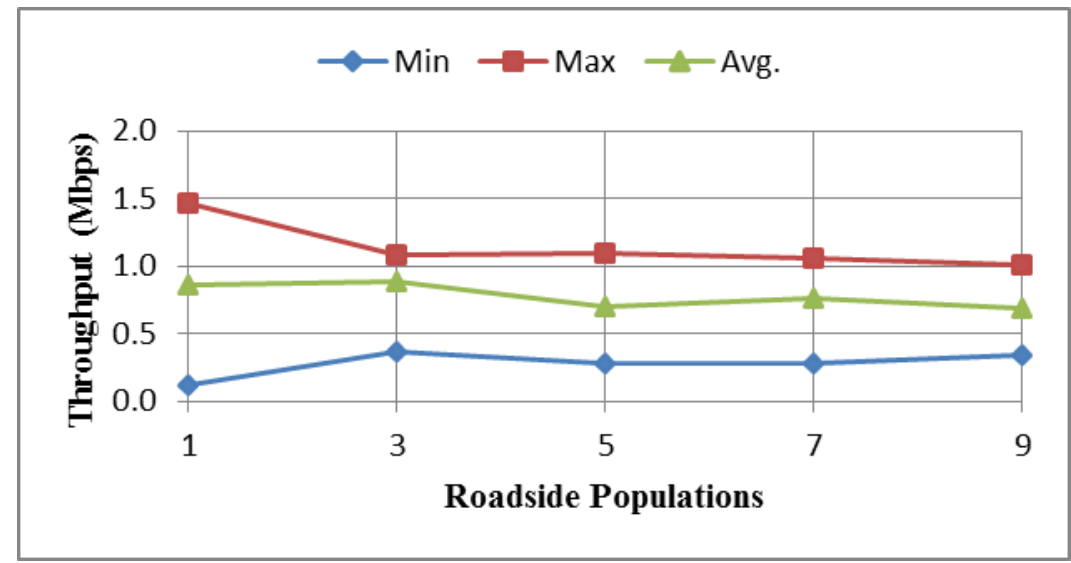

(a)

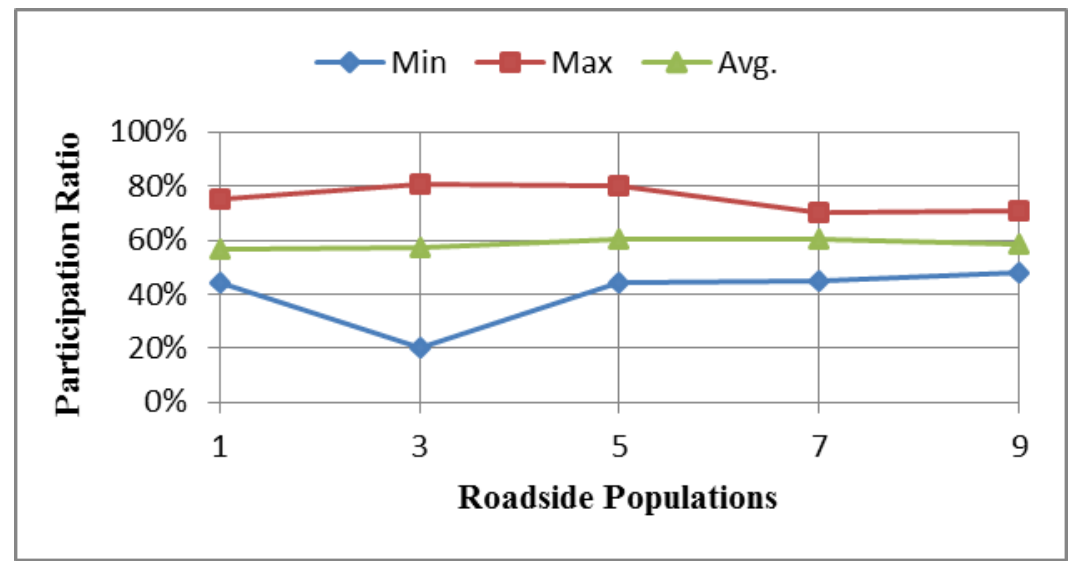

(b)

Figure 4. Experimental Results for 30 Vehicular Nodes

From Figure 4(a), the proposed algorithm attains an average data throughput of approximately $0.88 \mathrm{Mbps}$. The changeable roadside populations affect the data transfer proportion for the same vehicular density (i.e., 30 vehicular nodes). In addition, the roadside participation percentage decreases to $57.3 \%$ along with a reduction in the maximum percentage. Compared to the previous results for 10 and 20 vehicles respectively, the participation percentage can reach $100 \%$, while only $80.1 \%$ are 
achieved with the 30 vehicles. Based on Figure 5(a), the highest data throughput for 40 vehicles is $0.77 \mathrm{Mbps}$. The presence of 7 roadsides can support the requested vehicular nodes and can reach a throughput rate of $0.99 \mathrm{Mbps}$ in the maximum case. Figure 5(b) illustrates the roadside participation percentage for various roadside populations serving 40 vehicular nodes.

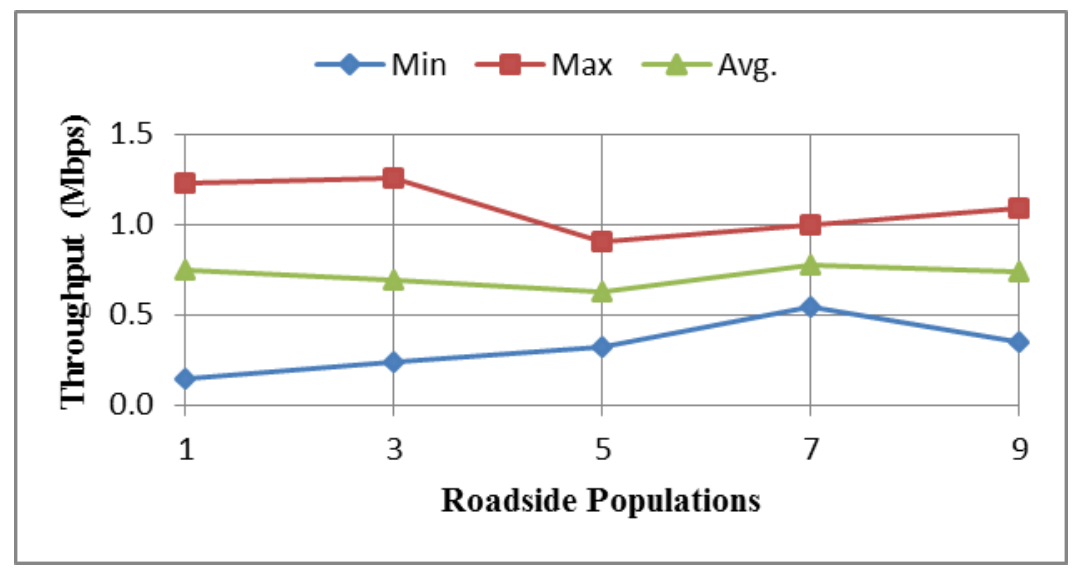

(a)

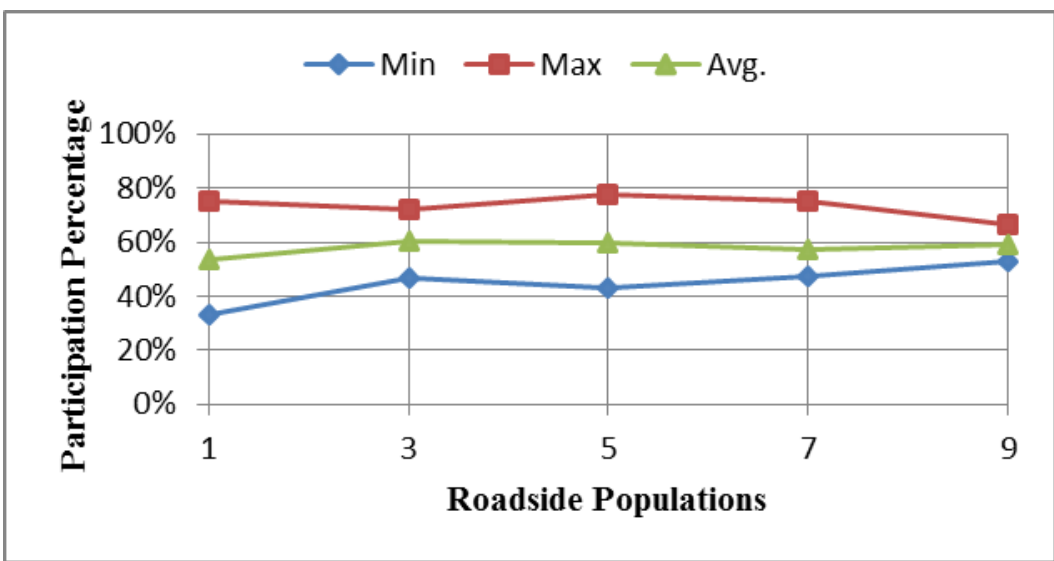

(b)

\section{Figure 5. The Numerical Results of Forming Coalitions Using 40 Vehicular Nodes}

Based on the conducted experiments, the highest average throughput recorded is 1.06 Mbps for 10 vehicular nodes. Additionally, the data throughput reduces as the vehicular densities increases using the same roadside population. This is because the network workload increases as long as the number of requesting vehicles increases.

\section{Conclusion}

Recently, VANETs is considered to be a promising subclass of Mobile Ad hoc Networks (MANETs) that constitutes the future of ITS. In this paper, we introduce a coalition formation algorithm to resolve the conflicting environment arise when demanding sharable resources. We investigate the impact of several roadside populations on the network throughput. We also study the roadside participation percentage using different vehicular densities divided into two regions. Vehicular nodes 
can form feasible coalitions in order to enhance the resource utilization. The conducted simulation results are achieved by executing 20 experiments. The highest average data throughput observed is $1.06 \mathrm{Mbps}$ for 10 vehicular nodes along with a maximum roadside participation percentage up to $100 \%$. Finally, issues including handling the channel radios and cooperation in inter-roadside communications are still a present challenge.

\section{Acknowledgements}

This work was supported by The National High-Tech Research and Development Plan of China under Grant No.2012AA01A301-0, and The National Natural Science Foundation of China (61173036).

\section{References}

[1] A. Boukerche, H. A. Oliveira, E. F. Nakamura, and A. A. Loureiro, "Vehicular ad hoc networks: A new challenge for localization-based systems," Computer communications, vol. 31, (2008), pp. 2838-2849.

[2] G. Samara, W. A. Al-Salihy, and R. Sures, "Security Analysis of Vehicular Ad Hoc Nerworks (VANET)," in Network Applications Protocols and Services (NETAPPS), 2010 Second International Conference on, (2010), pp. 55-60.

[3] H. Kawashima, "Japanese perspective of driver information systems," Transportation, vol. 17, (1990), pp. 263-284.

[4] Y. Toor, P. Muhlethaler, and A. Laouiti, "Vehicle ad hoc networks: Applications and related technical issues," Communications Surveys \& Tutorials, IEEE, vol. 10, (2008), pp. 74-88.

[5] S. Al-Sultan, M. M. Al-Doori, A. H. Al-Bayatti, and H. Zedan, "A comprehensive survey on vehicular Ad Hoc network," Journal of network and computer applications, vol. 37, (2014), pp. 380-392.

[6] H. Hartenstein and K. P. Laberteaux, "A tutorial survey on vehicular ad hoc networks," Communications Magazine, IEEE, vol. 46, (2008), pp. 164-171.

[7] S.-W. Wang and M.-Y. Chang, "Roadside units allocation algorithms for certificate update in vanet environments," in Communications (APCC), 2011 17th Asia-Pacific Conference on, (2011), pp. 472-477.

[8] A. Abdrabou and W. Zhuang, "Probabilistic delay control and road side unit placement for vehicular ad hoc networks with disrupted connectivity," Selected Areas in Communications, IEEE Journal on, vol. 29, (2011), pp. 129-139.

[9] J. Lee and C. M. Kim, "A roadside unit placement scheme for vehicular telematics networks," in Advances in Computer Science and Information Technology, ed: Springer, (2010), pp. 196-202.

[10] E. S. Cavalcante, A. L. Aquino, G. L. Pappa, and A. A. Loureiro, "Roadside unit deployment for information dissemination in a vanet: an evolutionary approach," in Proceedings of the fourteenth international conference on Genetic and evolutionary computation conference companion, (2012), pp. 27-34.

[11] D. S. Hochbaum, Approximation algorithms for NP-hard problems: PWS Publishing Co., (1996).

[12] D. Niyato, P. Wang, W. Saad, and A. Hjorungnes, "Coalition formation games for bandwidth sharing in vehicle-to-roadside communications," in Wireless Communications and Networking Conference (WCNC), 2010 IEEE, (2010), pp. 1-5.

[13] A. Chaintreau, P. Hui, J. Crowcroft, C. Diot, R. Gass, and J. Scott, "Impact of human mobility on opportunistic forwarding algorithms," Mobile Computing, IEEE Transactions on, vol. 6, (2007), pp. 606-620.

[14] R. B. Myerson, "Game theory: analysis of conflict", Cambridge: Mass, Harvard University, (1991).

[15] S. Hadzic, S. Mumtaz, and J. Rodriguez, "Cooperative Game Theory and Its Application in Localization Algorithms," GAME THEORY RELAUNCHED, (2013), p. 173.

[16] M. Barth and K. Boriboonsomsin, "ECO-ITS: Intelligent Transportation System Applications to Improve Environmental Performance," National Technical Information Service, No. FHWA-JPO-12-042, (2012).

[17] M.-F. Jhang and W. Liao, "Cooperative and opportunistic channel access for vehicle to roadside (V2R) communications," Mobile Networks and Applications, vol. 15, (2010), pp. 13-19.

[18] D. Niyato, Z. Han, W. Saad, and A. Hjorungnes, "A controlled coalitional game for wireless connection sharing and bandwidth allocation in mobile social networks," in Global Telecommunications Conference (GLOBECOM 2010), 2010 IEEE, (2010), pp. 1-5.

[19] S. Vassaki, A. D. Panagopoulos, and P. Constantinou, "Bandwidth allocation in wireless access networks: Bankruptcy game vs cooperative game," in Ultra Modern Telecommunications \& Workshops, 2009. ICUMT'09. International Conference on, (2009), pp. 1-4. 
[20] W. Chen, R. K. Guha, T. J. Kwon, J. Lee, and Y. Y. Hsu, "A survey and challenges in routing and data dissemination in vehicular ad hoc networks," Wireless Communications and Mobile Computing, vol. 11, (2011), pp. 787-795.

[21] B. P. Crow, I. Widjaja, J. G. Kim, and P. T. Sakai, "IEEE 802.11 wireless local area networks," Communications Magazine, IEEE, vol. 35, (1997), pp. 116-126.

\section{Authors}

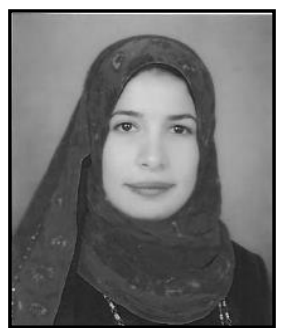

Esraa M. Eldesouky, she is a Ph.D. student in college of Information Science and Engineering at Hunan University. She earned the M.Sc. degree in computer science and technology from Hunan University (2011). She worked at Faculty of Computers and Informatics in Suez Canal University as a teaching assistant from 2005 to 2008. The current research interest includes VANETs, content handling, intelligent transportation systems, and coalition formation game.

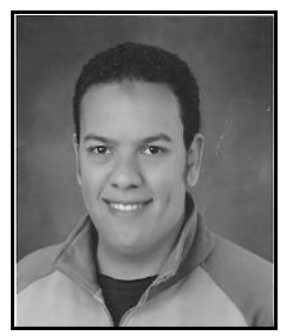

Ahmed M. Ali, he is a Ph.D. candidate at College of Information Science and Engineering, Hunan University. Earlier, he finished his M.Sc. degree in the same college at Hunan University in 2011. His research interests include mobile nodes, channel estimation and modulation, and VANETs simulation.

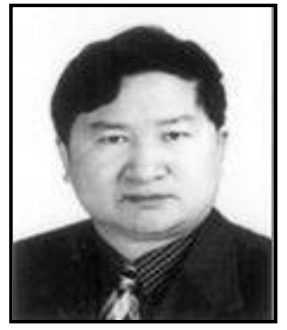

Li Renfa, he is a Professor in the college of Information Science and Engineering at Hunan University. He received the B.Eng. and M.Eng. degrees from Tianjin University, China in 1982 and 1987, and the Ph.D. degree from Huazhong University of Sciences and Technology, China in 2003. He was a Professor at Hunan Technology University from 1987 to 1999. From 2000, he became the dean at the college of Computer and Communication, Hunan University. His research interests are in the areas of embedded system architecture, cyber-physical system and wireless networks. $\mathrm{He}$ is the founder of Embedded Systems \& Networking Laboratory of Hunan University, and the leader of Hunan Provincial Key Laboratory of Network and Information Security of Hunan University.

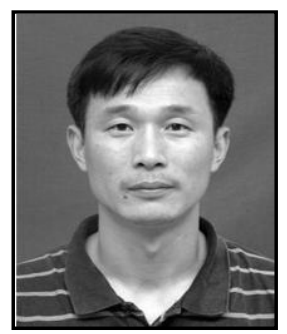

Wang Dong, born in 1964. professor, Ph.D. supervisor of Hunan University. Senior member of China Computer Federation. He has visited University Technology of Sydney, Sydney, Australian from 2004.12 to 2005.12,His current research interests include network test and performance evaluation, wireless communications and mobile computing, VANET etc. He had published more than 70 journal papers. 
International Journal of Hybrid Information Technology Vol. 7, No. 6 (2014) 